\title{
Out-of-equilibrium dynamics of multiple second-order quantum phase transitions in extended Bose-Hubbard model: Superfluid, supersolid and density wave
}

\author{
Keita Shimizu ${ }^{1}$, Takahiro Hirano ${ }^{1}$, Jonghoon Park $^{1}$, Yoshihito Kuno ${ }^{2}$, and Ikuo Ichinose ${ }^{1}$ \\ ${ }^{1}$ Department of Applied Physics, Nagoya Institute of Technology, Nagoya, 466-8555, Japan and \\ ${ }^{2}$ Department of Physics, Graduate School of Science, Kyoto University, Kyoto, 606-8502, Japan
}

(Dated: November 15, 2018)

\begin{abstract}
In this paper, we study the dynamics of the Bose-Hubbard model with the nearest-neighbor repulsion by using time-dependent Gutzwiller methods. Near the unit filling, the phase diagram of the model contains density wave (DW), supersolid (SS) and superfluid (SF). The three phases are separated by two second-order phase transitions. We study "slow-quench" dynamics by varying the hopping parameter in the Hamiltonian as a function of time. In the phase transitions from the DW to SS and from the DW to SF, we focus on how the SF order forms and study scaling laws of the SF correlation length, vortex density, etc. The results are compared with the Kibble-Zurek scaling. On the other hand from the SF to DW, we study how the DW order evolves with generation of the domain walls and vortices. Measurement of first-order SF coherence reveals interesting behavior in the DW regime.

PACS numbers: $\quad 67.85 . \mathrm{Hj}, 03.75 . \mathrm{Kk}, 05.30 . \mathrm{Rt}$
\end{abstract}

\section{INTRODUCTION}

Systems of ultra-cold atomic gases have the high versatility and controllability. In the last decades, ultra-cold atomic gas systems play an important role for the study on the quantum many physics as quantum simulators [15]. In this paper, we study ultra-cold Bose gas systems as a quantum simulator for out-of-equilibrium dynamics of many-body quantum systems. For a finite-temperature quench, from the view point of the cosmology, Kibble [6, 7] studied how the system exhibits out-of-equilibrium behavior and pointed out that the phase transitions lead to topological defects as a result of spontaneous symmetry breaking of continuous symmetries. After the pioneering work by Kibble, Zurek [8 10] found that a similar phenomenon is to be observed in experiments on the condensed matter systems such as the superfluid (SF) of ${ }^{4} \mathrm{He}$. Furthermore for the second-order phase transition, it was argued that physical quantities satisfy some kind of scaling laws with respect to the quench time that measures the speed of the "slow quench". The works by Kibble and Zurek stimulated many physicists, and there appeared many theoretical and experimental studies to test this conjecture, which is sometimes called Kibble-Zurek (KZ) mechanism and KZ scaling [11]. Recent experiments on ultra-cold atomic gases in a homogeneous density setup verified the KZ scaling law for the correlation length and topological defect formation [12, 13].

Similar problem was also studied for quantum systems, i.e., how low-energy states evolve under a change of the parameters in the Hamiltonian crossing a quantum phase transition (QPT), i.e., the quantum quench [14 20]. This problem has also attracted great interests. Experiments on behaviors of quantum systems through QPTs have been already done using the ultra-cold atomic gases as a quantum simulator 21 25].

In the previous two papers [26, 27], we study the outof-equilibrium dynamics of the ultra-cold Bose atoms on a square optical lattice by using the Bose-Hubbard models. In the practical calculation, we fixed the on-site and nearest-neighbor (NN) repulsions and varied the hopping amplitude in the Hamiltonian, and studied how the lowest-energy state evolves. In Ref. [26], we investigated how the ground state evolves from the Mott insulator to SF by means of the time-dependent Gutzwiller (GW) methods. We first showed the behavior of the SF order parameter, and gave physical pictures of the out-ofequilibrium behavior of the system. We found that the physical quantities such as the correlation length of the SF, vortex density, etc. satisfy scaling laws and compared the obtained scaling exponents with the predicted values via the $\mathrm{KZ}$ hypothesis. On the other hand in Ref. [27], we considered an extended Bose-Hubbard model, which includes the NN repulsion. Phase diagram has the SF, density wave (DW) and also the supersolid (SS). For fairly weak NN repulsion, there exists a first-order phase transition directly connecting the DW and SF phases accompanying a finite jump in physical quantities [28, 29]. We focused on that parameter regime, and studied the quench dynamics from the DW to SF, and vice-versa.

In this work, we consider the intermediate strength of the NN repulsion. In this parameter regime, there exist two second-order phase transitions separating the DW and SS, and also the SS and SF [28, 29]. Therefore, out-of-equilibrium dynamics of the multiple phase transitions can be studied. There are two out-of-equilibrium 'impulse' regimes in the quench dynamics, and their locations are rather close with each other. Then, it is interesting to see if scaling laws similar to the $\mathrm{KZ}$ hold or not, how the existence of the intermediate SS changes the quench dynamics of the DW and SF, etc [30].

This paper is organized as follows. In Sec. II, we introduce the extended Bose-Hubbard model on the square lattice, and define order parameters used to distinguish various phases. Equilibrium phase diagram obtained by the static GW methods is shown. There are three phases, 
i.e., DW, SS and SF.

In Sec. III, we show the results of the quench dynamics from the DW to SS, and also from the DW to SF through the SS. We study the behavior of the SF order parameter in detail and see if scaling laws of the SF correlation length, etc, hold. The results are compared with the KZ mechanism, and estimation of the critical exponents is given. We also calculate the SF correlation length in the SF regime and examine what kind of state forms there.

In Sec. IV, we study quench dynamics from the SF to DW through SS. Behavior of the SF order parameter depends on the quench time $\tau_{\mathrm{Q}}$. For small $\tau_{\mathrm{Q}}$ (fast quench), domain walls of finite-size DWs form and the amplitude of the SF remains finite. We also show that quantum vortices are bound on domain walls. On the other hand for large $\tau_{\mathrm{Q}}$ (slow quench), individual DW region is large. However, the first-order correlation of the boson operator has a peculiar behavior. Its origin is discussed.

Section V is devoted for conclusion and discussion.

\section{EXTENDED BOSE-HUBBARD MODEL AND EQUILIBRIUM PHASE DIAGRAM}

We consider the two-dimensional extended BoseHubbard model (EBHM) described by the following Hamiltonian,

$$
\begin{aligned}
H_{\mathrm{EBH}}= & -J \sum_{\langle i, j\rangle}\left(a_{i}^{\dagger} a_{j}+\text { H.c. }\right)+\frac{U}{2} \sum_{i} n_{i}\left(n_{i}-1\right) \\
& +V \sum_{\langle i, j\rangle} n_{i} n_{j}-\mu \sum_{i} n_{i},
\end{aligned}
$$

where $\langle i, j\rangle$ denotes a pair of NN sites of a square lattice, $a_{i}^{\dagger}\left(a_{i}\right)$ is the creation (annihilation) operator of boson at site $i$, and $n_{i}=a_{i}^{\dagger} a_{i} . J$ is the hopping amplitude, and $\mu$ is the chemical potential. There are two kind of repulsions in the model, i.e., $U$ and $V$-terms in Eq.(11), which describe the on-site and NN repulsions, respectively. For $J, V<U$, the system is in the Mott insulator, whereas for $J>U, V$, the SF forms. On the other hand for $V>J, U$, the DW order is realized. As we see later on, there exists another phase, i.e., SS, which has both the DW and SF orders.

In this paper, we consider the system near the unit filling $\rho=\frac{1}{N_{s}} \sum_{i}\left\langle n_{i}\right\rangle=1$, where $N_{s}$ is the number of lattice sites. In most of the practical calculations, we set $N_{s}=64 \times 64$ with the periodic boundary condition. In the previous work [27], we focused on the system near the half filling $\rho=1 / 2$ and weak $\mathrm{NN}$ repulsion such as $V / U=0.05$, and studied the first-order phase transition between the DW and SF. On the other hand in this work, we consider the near unit filling case $\rho \approx 1$ and relatively large $V$, and study the phase transitions including the DW, SS and SF.

In the present work, we study quench dynamics of the system of $H_{\mathrm{EBH}}$. To this end, we employ the timedependent GW (tGW) methods 31 37]. The tGW meth- ods approximate the Hamiltonian of the EBHM in Eq.(1) with a single-site Hamiltonian $H_{i}$ by introducing local expectation value $\Psi_{i}=\left\langle a_{i}\right\rangle$,

$$
\begin{aligned}
H_{\mathrm{GW}}= & \sum_{i} H_{i}, \\
H_{i}= & -J \sum_{j \in i \mathrm{NN}}\left(a_{i}^{\dagger} \Psi_{j}+\text { H.c. }\right)+\frac{U}{2} n_{i}\left(n_{i}-1\right) \\
& +V \sum_{j \in i \mathrm{NN}} n_{i}\left\langle n_{j}\right\rangle-\mu n_{i},
\end{aligned}
$$

where $i \mathrm{NN}$ denotes the NN sites of site $i$ and for NN repulsion term the Hartree-Fock decoupling has been introduced. To solve the quantum system $H_{\mathrm{GW}}$ in Eq.(2), we introduce the following site-factorized wave function,

$$
\left|\Phi_{\mathrm{GW}}\right\rangle=\prod_{i}^{N_{s}}\left(\sum_{n=0}^{n_{c}} f_{n}^{i}(t)|n\rangle_{i}\right), \quad a_{i}^{\dagger} a_{i}|n\rangle_{i}=n|n\rangle_{i},
$$

where $n_{c}$ is the maximum number of particle at each site, and we take $n_{c}=6$ in the present work. In terms of $\left\{f_{n}^{i}(t)\right\}$, the SF order parameter is given as,

$$
\Psi_{i}=\left\langle a_{i}\right\rangle=\sum_{n=1}^{n_{c}} \sqrt{n} f_{n-1}^{i *} f_{n}^{i},
$$

and $\left\{f_{n}^{i}(t)\right\}$ are determined by solving the following Schrödinger equation for various initial states,

$$
i \hbar \partial_{t}\left|\Phi_{\mathrm{GW}}\right\rangle=H_{\mathrm{GW}}(t)\left|\Phi_{\mathrm{GW}}\right\rangle .
$$

The time dependence of $H_{\mathrm{GW}}(t)$ in Eq. (5) comes from the quench $J \rightarrow J(t)$ with fixed $U$ and $V$ as explained in the following section. We employ the fourth-order RungeKutta method for study of the time evolution in Eq.(5). In the practical calculation, we prepare 10 states as the initial state and study the time evolution of each state. Physical quantities are obtained by averaging results of the time evolutions with 10 initial states. Applicability and reliability of the GW methods are discussed rather in detail in Ref. [27].

In order to obtain the phase diagram, we calculate the following order parameters to distinguish the above mentioned states, i.e., the DW, SF and SS,

$$
\begin{aligned}
& \Psi_{i}=\left\langle a_{i}\right\rangle, \quad|\Psi|=\frac{1}{N_{s}} \sum_{i}\left|\Psi_{i}\right|, \\
& \Delta_{\mathrm{DW}}=\frac{1}{N_{s}} \sum_{i}(-1)^{i}\left\langle n_{i}\right\rangle, \\
& \Delta_{\mathrm{SF}}=\frac{1}{N_{s}} \sum_{i}(-1)^{i}\left|\Psi_{i}\right|,
\end{aligned}
$$

where $(-1)^{i}$ stands for $+1(-1)$ for even sites (odd sites).

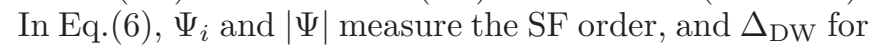
the DW, whereas a finite $\Delta_{\mathrm{SF}}$ indicates the existence of the SS, and $\Delta_{\mathrm{SF}}$ is called relative order parameter [38]. In the study of the non-equilibrium quench dynamics, 

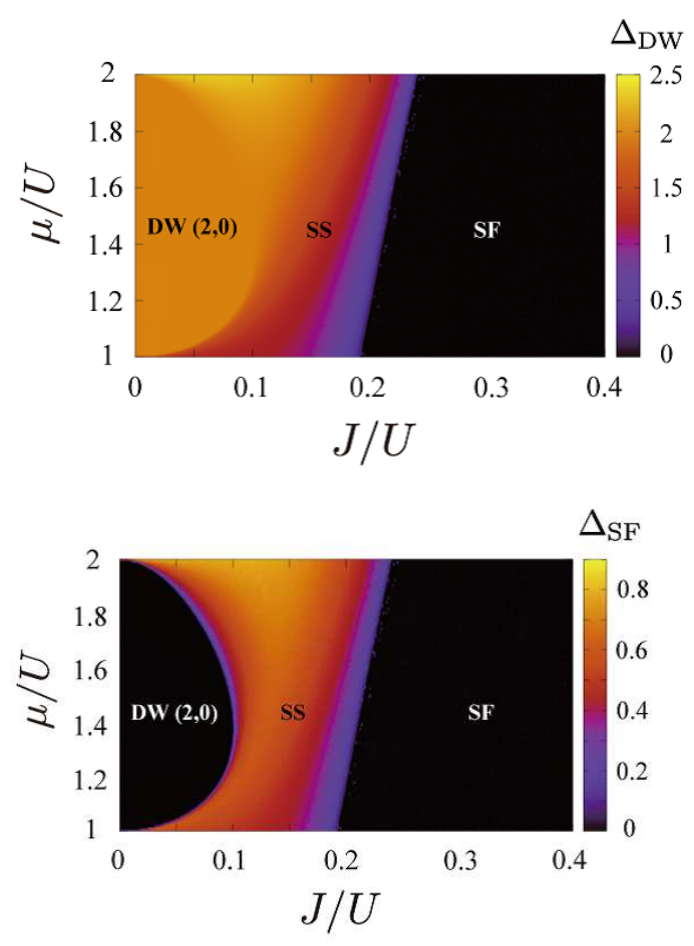

FIG. 1. Phase diagrams of the EBHM near the unit filling and $V / U=0.375$. There are three phases, (2,0)-type DW, SS and SF. These phases are separated by the second-order phase transitions. In the DW, $\Delta_{\mathrm{DW}} \neq 0, \Delta_{\mathrm{SF}}=0,|\Psi|=0$. On the other hand in the SS, $\Delta_{\mathrm{DW}} \neq 0, \Delta_{\mathrm{SF}} \neq 0,|\Psi| \neq 0$. In the SF, $\Delta_{\mathrm{DW}}=0, \Delta_{\mathrm{SF}}=0,|\Psi| \neq 0$.

the above quantities play an important role and they are measured as a function of time.

Before going into the out-of-equilibrium dynamics of the system, we show the equilibrium phase diagram of the EBHM. To this end, we solve the time-independent Schrödinger equation for the Hamiltonian $H_{\mathrm{GW}}$. We show the obtained phase diagram for $V / U=0.375$ in Fig. 1 and the physical quantities in Fig. 2, which are calculated by the static GW wave functions and used for identification of phases. There are three phases for $V / U=0.375$, i.e., the SF, DW and SS. The SS has both the SF and DW order, and is located between the SF and DW in the phase diagram. There are two phase boundaries, and both phase transitions are of second order as the physical quantities in Fig. 2 indicate. This result is in good agreement with that of the previous study using quantum Monte-Carlo (QMC) simulations [29] although the obtained region of the SS phase is slightly larger than the QMC results.

In the subsequent sections, based on the phase diagram in Fig. 1 we shall study out-of-equilibrium quench dynamics of the system that takes place when the system crosses the phase boundaries as a result of temporal change in parameters in the Hamiltonian in Eq.(2). In the practical calculation, we fix $U=1$ as the unit of en-

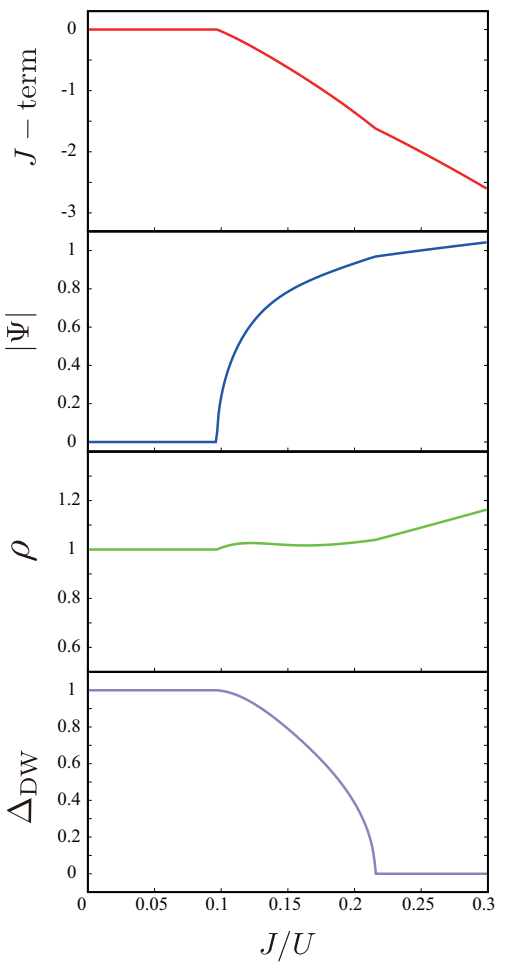

FIG. 2. Calculations of the physical quantities for the phase diagrams in Fig. 1 Chemical potential $\mu / U=1.5$ and $V / U=$ 0.375. $J$-term stands for the expectation value of the hopping term $-\sum_{\langle i, j\rangle}\left(a_{i}^{\dagger} a_{j}+\right.$ H.c. $) .|\Psi|$ and $\Delta_{\text {DW }}$ are order parameters of the $\mathrm{SF}$ and DW, respectively. $\rho$ is the mean particle density. Phase transitions take place at $J / U=J_{c 1} / U \simeq 0.10$, and $J / U=J_{c 2} / U \simeq 0.22$.

ergy, and also we focus on the case with $V / U=0.375$ as in the static case. In the previous work [27], we studied the system with $V / U=0.05$, in which the SS does not forms near $\rho \approx 0.5$ and a first-order phase boundary exists between the SF and DW. In the present work, we are interested in how the system evolves when it crosses the multiple second-order phase transitions, etc.

\section{TRANSITIONS FROM DW TO SS AND FROM DW TO SF}

In this section, we consider the dynamics of the transitions from the DW to SS and from the DW to SF. As shown in Fig. 3, the mean particle density $\rho \approx 1$ for $\mu / U=1.5$, and then the DW is the $(2,0)$-type one. Phase transition from the DW to SS takes place at $J / U=J_{c 1} / U \simeq 0.10$, and from the $\mathrm{SS}$ to $\mathrm{SF}$ at $J / U=J_{c 2} / U \simeq 0.22$, respectively. 


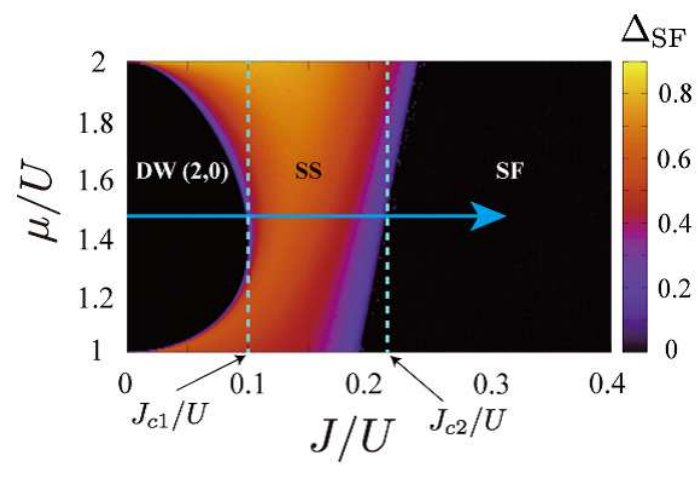

FIG. 3. Arrow indicates quench protocol from the DW to SS and $\mathrm{SF}$ in the phase diagram in Fig. 1. For $\mu / U=1.5$, the critical points are located at $J_{c 1} / U=0.10$ and $J_{c 2} / U=0.22$.
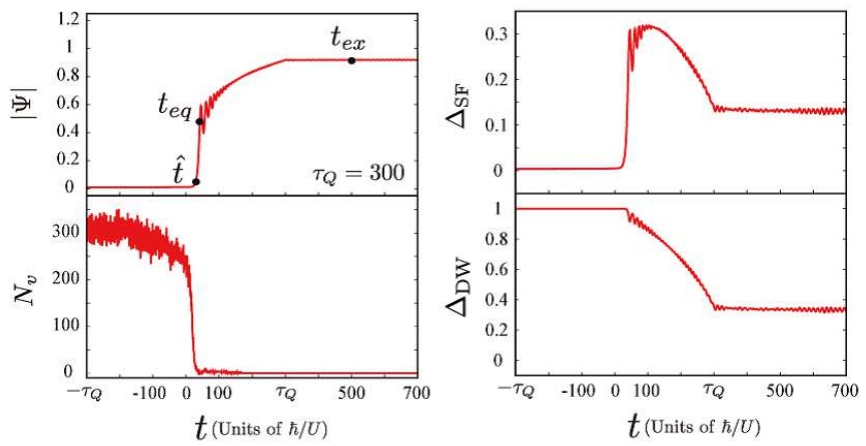

FIG. 4. Calculations of the physical quantities from the DW to SS as a function of time. At $t=0$, the system passes trough the equilibrium phase transition point DW $\rightarrow$ SS. Locations of $\hat{t}, t_{\text {eq }}$ and $t_{\text {ex }}$ are indicated.

\section{A. From DW to SS}

We study the transition from the DW to SS first. In the practical calculation, the following quench protocol is used;

$$
\frac{J(t)-J_{c 1}}{J_{c 1}}=\frac{t}{\tau_{\mathrm{Q}}}, \quad t \in\left[-\tau_{\mathrm{Q}}, \tau_{\mathrm{Q}}\right],
$$

where $\tau_{\mathrm{Q}}$ is called quench time. The protocol in Eq.(7) indicates that the system crosses the equilibrium phase transition point $J_{c 1}$ at $t=0$, and the quench terminates at $t=\tau_{\mathrm{Q}}$ with $J\left(\tau_{\mathrm{Q}}\right)=2 J_{c 1}\left(<J_{c 2}\right)$.

In Fig. 4, we show the order parameters $|\Psi|, \Delta_{\mathrm{DW}}$, and $\Delta_{\mathrm{SF}}$ as a function of time $(t)$ for $\tau_{\mathrm{Q}}=300$. $|\Psi|$ exhibits a similar behavior to that in the transition from the Mott to $\mathrm{SF}$ in the $V / U=0$ case studied previously 26]. As in the previous works, we define the transition time $\hat{t}$, at which the system evolves from the impulse to adiabatic regimes, by $|\Psi(\hat{t})|=2|\Psi(0)|[39]$. On the other hand, $t_{\text {eq }}$ is the time at which the oscillating behavior of $|\Psi|$ starts. Physical picture of the oscillating regime was explained
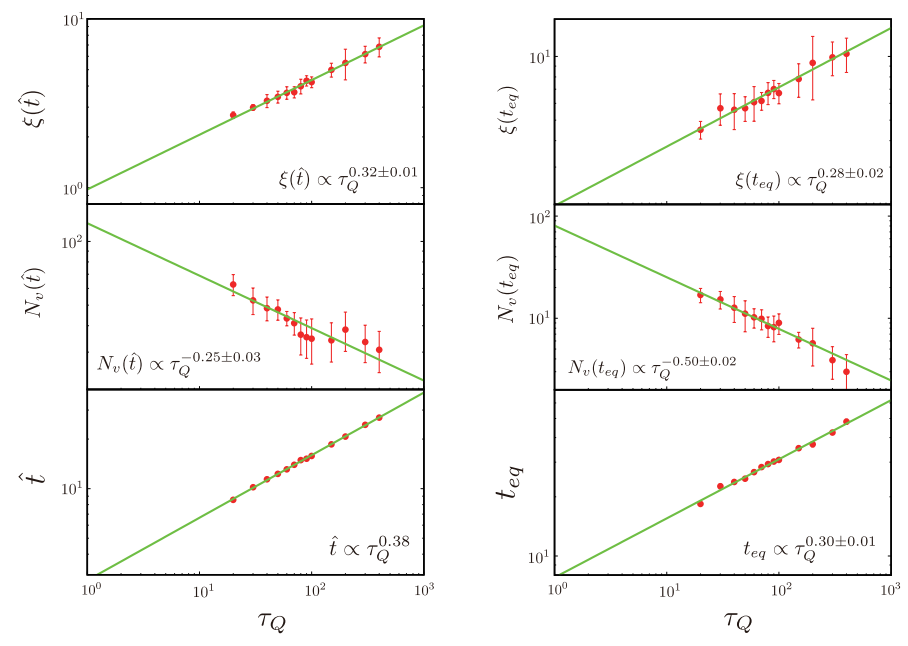

FIG. 5. Observation of scaling laws with respect to $\tau_{\mathrm{Q}}$ for various quantities at $t=\hat{t}$ and $t=t_{\mathrm{eq}}$. The exponents are indicated with errors. The error of the exponent in the bottomleft caption is smaller than 0.01 .

in the previous paper [26]. The amplitude of $\mathrm{SF},|\Psi|$, develops quite rapidly from $\hat{t}$ to $t_{\mathrm{eq}}$. On other hand, the correlation length only doubles in that period. Genuine coarsening process of the long-range SF coherence takes place between $t_{\mathrm{eq}}$ and $t_{\mathrm{ex}}$, where $t_{\mathrm{ex}}$ is the time at which the oscillation of $|\Psi|$ terminates.

The other order parameters, $\Delta_{\mathrm{DW}}, \Delta_{\mathrm{SF}}$ and $N_{v}$ [defined by Eq.(8)] in Fig. [4 show that the system evolves into the SS at $t=\hat{t}(=23.5)$. This result indicates that the present definition of $\hat{t}$ is a suitable one, that is, the adiabatic development of the SF order starts at $t=\hat{t}$.

It is quite interesting and important to see if scaling laws of physical quantities, such as the SF correlation length and vortex density, with respect to the quench time $\tau_{\mathrm{Q}}$ hold or not. Here, the SF correlation length, $\xi$, and vortex density, $N_{v}$, are defined as

$$
\begin{aligned}
& \frac{1}{8 N_{s}} \sum_{i}\left\langle a_{i}^{\dagger} a_{i \pm r \hat{x}(\hat{y})}+\text { H.c. }\right\rangle \propto \exp (-r / \xi), \quad(r \gg 1) \\
& N_{v}=\sum_{i}\left|\Omega_{i}\right|, \\
& \Omega_{i}=\frac{1}{4}\left[\sin \left(\theta_{i+\hat{x}}-\theta_{i}\right)+\sin \left(\theta_{i+\hat{x}+\hat{y}}-\theta_{i+\hat{x}}\right)\right. \\
& \left.\quad \quad-\sin \left(\theta_{i+\hat{x}+\hat{y}}-\theta_{i+\hat{y}}\right)-\sin \left(\theta_{i+\hat{y}}-\theta_{i}\right)\right],
\end{aligned}
$$

where $\theta_{i}$ is the phase of $\Psi_{i}$ and $\hat{x}(\hat{y})$ is the unit vector in the $x(y)$ direction. As the transition from the DW to SS is of second-order, one may expect that the correlation length and the vortex density satisfy a scaling law with the critical exponents of the 3D XY model, which describes the second-order SF phase transition.

To see the relation between the Bose-Hubbard model and the 3D XY model, the path-integral quantization is useful [40]. By introducing the time $t$, and complex fields 
$\psi_{i}$ and $\bar{\psi}_{i}$ for the operators $a_{i}$ and $a_{i}^{\dagger}$, respectively, the time evolution of the system is given by the following path integral,

$$
\int[d \psi] \exp \left[\int d t\left(-\sum_{i} \bar{\psi}_{i} \partial_{t} \psi_{i}-i H(\bar{\psi}, \psi)\right)\right]
$$

where $H(\bar{\psi}, \psi)$ is the Bose-Hubbard Hamiltonian with the $J$ and $U$-terms. In the SF critical region, density fluctuations are small and the phase degrees of freedom $\left\{\theta_{i}\right\}$ play an important role. Therefore, we put $\psi_{i}=$ $\sqrt{\rho_{i}} e^{i \theta_{i}}$, and expand as $\rho_{i}=\rho_{0}+\delta \rho_{i}$, where $\rho_{0}$ is the mean density controlled by the chemical potential. (We use the same notation $\theta_{i}$ as in Eq. (8), for it essentially refers to the same thing.) In Eq. (9),

$$
\begin{aligned}
& -\sum_{i} \bar{\psi}_{i} \partial_{t} \psi_{i}-i H(\bar{\psi}, \psi) \\
& \rightarrow-i \sum_{i} \delta \rho_{i} \partial_{t} \theta_{i}+i J \rho_{0} \sum_{\langle i, j\rangle}\left(e^{-i \theta_{i}} e^{i \theta_{j}}+\text { c.c. }\right) \\
& \quad-i U \sum_{i}\left(\delta \rho_{i}\right)^{2} .
\end{aligned}
$$

Integration over $\delta \rho_{i}$ can be readily performed as follows,

$$
\begin{gathered}
\int d \delta \rho_{i} \exp \left[-i \int d t\left(\delta \rho_{i} \partial_{t} \theta_{i}+U\left(\delta \rho_{i}\right)^{2}\right)\right] \\
=e^{\frac{i}{4 U} \int d t\left(\partial_{t} \theta_{i}\right)^{2}} .
\end{gathered}
$$

Then, the resultant effective model describing the SF transition in the Bose-Hubbard model is given by the summation of the second $J$-term of Eq. (10) and the time-derivative term, $\sum_{i}\left(\partial_{t} \theta_{i}\right)^{2}=\sum_{i}\left(\partial_{t} e^{-i \theta_{i}} \cdot \partial_{t} e^{i \theta_{i}}\right)$, in Eq. (11). By introducing finite slices for the time direction, the 3D XY model is realized. The critical exponent of the spatial correlation length is given by the exponent of the 3D XY model, $\nu$. Furthermore, the dynamical exponent $z=1$ as the present 3D XY model describes (2Dspace $+1 \mathrm{D}$-time) dynamics symmetrically, and therefore the temporal correlation length $\xi_{t}$ is proportional to the spatial correlation length $\xi$.

However, it is not obvious that the above derivation of the 3D XY model is applicable for the present EBHM with the NN repulsion. Furthermore, the DW and SS are not homogeneous and also there exists the NN repulsions, and then a simple relation between the exponents such as $d=2 b$ may not hold, where exponent $b$ for $\xi \propto \tau_{\mathrm{Q}}^{b}$, and $d$ for $N_{v} \propto \tau_{\mathrm{Q}}^{-d}$. The above problems should be examined by the practical numerical calculations in the present work.

We show the obtained results in Fig. 15 for both $t=\hat{t}$ and $t=t_{\mathrm{eq}}$. It is obvious that $\xi$ and $N_{v}$ both satisfy a fairly good scaling law from $\tau_{\mathrm{Q}}=20$ to $\tau_{\mathrm{Q}}=400$. Exponents are estimated as $b=0.32$ and $d=0.25$ for $t=\hat{t}$, and $b=0.28$ and $d=0.50$ for $t=t_{\text {eq }}$, respectively. The vortex density at $t=t_{\mathrm{eq}}$ is smaller compared to that at $t=\hat{t}$. Then, the interactions between vortices are less effective at $t=t_{\mathrm{eq}}$, and as a result, the expected relation $d \approx 2 b$ holds for $t=t_{\text {eq }}$.
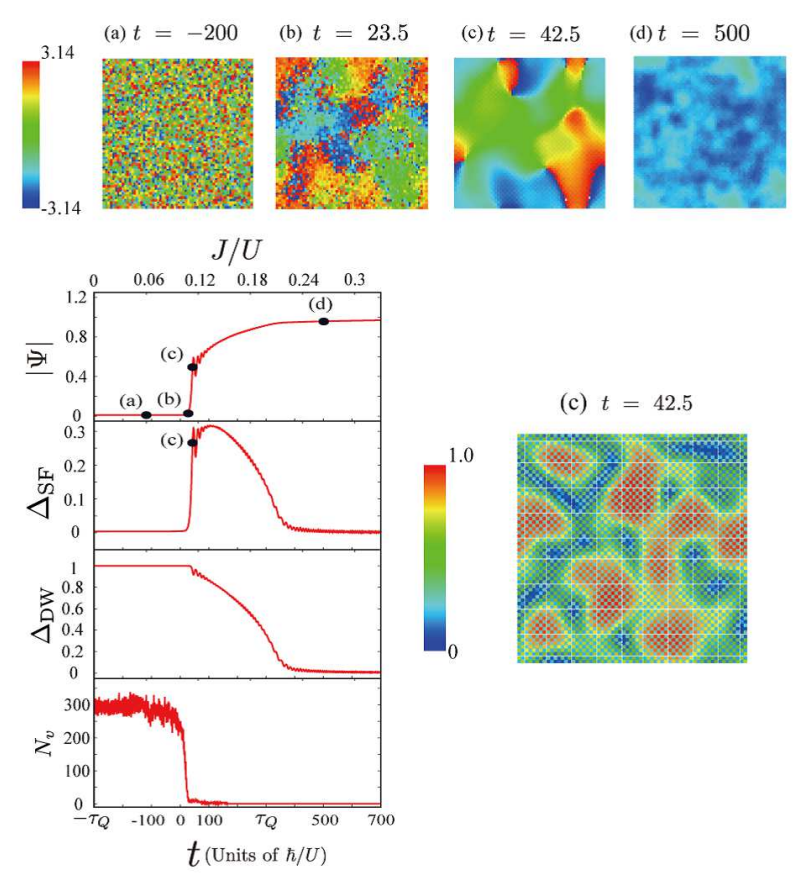

FIG. 6. Slow quench from the DW to SF through the SS. Physical quantities as a function of time for $\tau_{\mathrm{Q}}=300$. $t=$ 23.5 corresponds to $\hat{t}, t=42.5$ to $t_{\text {eq }}$ and $t=500$ to $t_{\text {ex }}$. The upper panels show phase of $\Psi_{i}$ for various times. Lowerright panel shows a profile of $\left|\Psi_{i}\right|$ in the SS. The system pass through $J=J_{c 1}\left(J_{c 2}\right)$ at $t=0\left(t=1.2 \tau_{\mathrm{Q}}\right)$.

We also show the scaling of $\hat{t}$ and $t_{\text {eq }}$ with respect to $\tau_{\mathrm{Q}}$ in Fig. 5 . For a second-order phase transition with the correlation-length exponent $\nu$ and dynamical exponent $z$, the KZ hypothesis predicts $\hat{t}, t_{\mathrm{eq}} \propto \tau_{\mathrm{Q}}^{\nu z / 1+\nu z}$ and $\xi \propto$ $\tau_{\mathrm{Q}}^{\nu / 1+\nu z}$. From the above results, we can estimate the critical exponents $\nu$ and $z$ as $\nu=0.51, z=1.18$ from the data at $\hat{t}$, and $\nu=0.40, z=1.07$ from the data at $t_{\mathrm{eq}}$, respectively. The estimated values of $z$ are fairly close to that expected from the 3D XY model, i.e., $z=1$. On the other hand, the estimated values of $\nu$ do not coincide with that of the 3D XY model, $\nu=0.672$ [41]. This may imply that the DW-type inhomogeneity influences the critical behavior of the SF order.

Finally in the above calculation, we have checked that the exponents, which we extract, are not sensitive to the exact definition of $\hat{t}$. In other words, in the period between $\hat{t}$ and $t_{\mathrm{eq}}, \xi$ and $N_{v}$ satisfy the scaling law quite well with the exponent close to that of $\hat{t}$ and $t_{\text {eq }}$. This result implies that SF droplets develop without collapsing with each other in that period [26, 27]. 


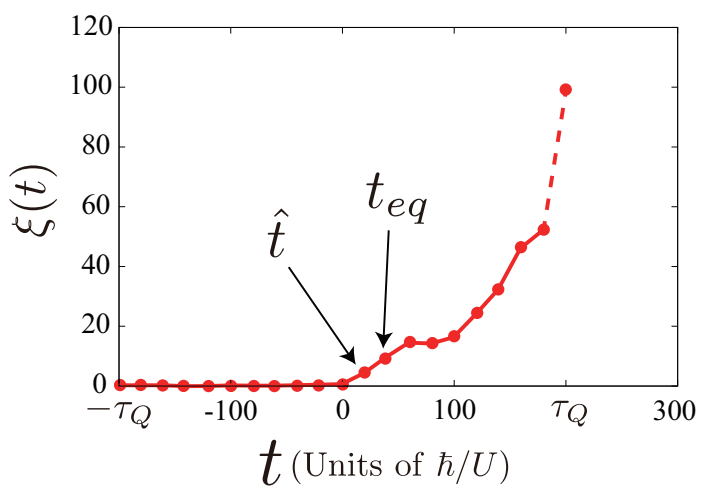

FIG. 7. SF correlation length as a function of time. $\tau_{\mathrm{Q}}=200$. After $t=0, \xi(t)$ increases quite rapidly. (The dotted line indicates the portion in which the correlation lengths exceed the system size.) This result indicates that a SF at finite temperature forms there.

\section{B. From DW to SF}

Let us turn to the case from the DW to SF through the SS. Quench protocol is as follows,

$$
\frac{J(t)-J_{c 1}}{J_{c 1}}=\frac{t}{\tau_{\mathrm{Q}}}, \quad t \in\left[-\tau_{\mathrm{Q}}, t_{f}\right]
$$

where we take the quench-termination time $t_{f}=700$ for the case of $\tau_{\mathrm{Q}}=300$. In Fig. 6. we show the behaviors of $|\Psi|, \Delta_{\mathrm{DW}}, \Delta_{\mathrm{SF}}$ and $N_{v}$ as a function of time. We also show snapshots of the phase of $\Psi_{i}$ in Fig. 6 (the upper panels). The DW order parameter decreases smoothly with small oscillations after the system passes the point $J / U \approx 0.1$, whereas the SF order parameter increases very rapidly after $\hat{t}$, and the coarsening process of the phase of $\Psi_{i}$ takes place smoothly from $t_{\mathrm{eq}}$ to $t_{\mathrm{ex}}$. Order parameter $\Delta_{\mathrm{SF}}$ has nonvanishing values only in the SS. Calculations in Fig. 6 show that the quench dynamics from the SS to SF is rather smooth compared with the DW to SS. Phase coarsening process of the SF order in the SS and SF accompanies fluctuations of the SF amplitude as discussed in the previous work [26].

It is interesting to see how the correlation length evolves under the quench, in particular, after the second critical point $J_{c 2}$. The result is shown in Fig. 7 From $\hat{t}$ to $t_{\text {eq }}$, the correlation length doubles, whereas it increases rapidly after $t_{\mathrm{eq}}$ as a result of the coarsening process of the phase degrees of freedom of $\Psi_{i}$. The calculation in Fig. 7 suggests that the correlation length diverges for large $t$. This result indicates that a homogeneous SF state at a finite temperature forms in that regime and it has a divergent Kosterlitz-Thouless type correlation length, i.e., the quench of the hopping amplitude injects energy into the system, and an equilibrium finite-temperature SF state is realized as a result.

Here, it is suitable to comment on the definition of $\hat{t}$. We employ its definition given in Ref. [39]. Our numeri- cal results in Fig. [ (in particular, the upper panels) and Fig. 7 exhibit that the adiabatic development of the SF order starts at $t=\hat{t}$. In fact, the phase of the SF order parameter acquires coherence at $t=\hat{t}$ as shown in Fig. 6. The correlation length of the SF order also starts to increase at $t=\hat{t}$. Therefore, it is suitable to think that a non-adiabatic chaotic state terminates at $t=\hat{t}$. On the other hand, the definition of $t_{\mathrm{eq}}$ is directly given by the behavior of $|\Psi|$ for each $\tau_{\mathrm{Q}}$. In Ref. [26], we discuss that the genuine coarsening process of local SF domains (bubbles) starts at $t_{\mathrm{eq}}$ [27], although more precise study of the coarsening process is desired.

\section{TRANSITION FROM SF TO DW}

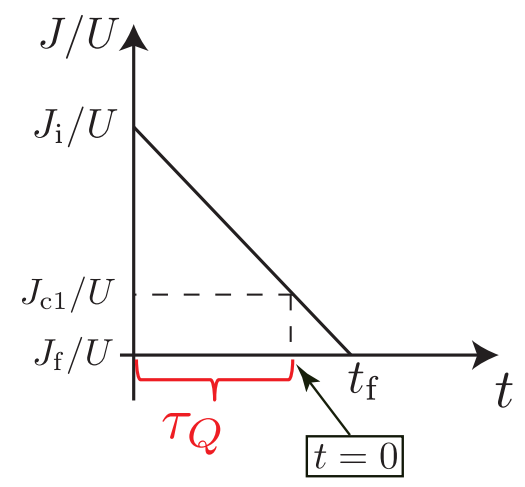

FIG. 8. Quench protocol of out-of-equilibrium dynamics in the precess $\mathrm{SF} \rightarrow \mathrm{SS} \rightarrow \mathrm{DW} .\left(J_{i} / U\right)=0.3$ and $\left(J_{f} / U\right)=0$.

In this section, we shall study dynamical behavior of the EBHM under the quench from the SF to DW. In the previous paper, we studied a related problem concerning to the first-order phase transition from the SF to the DW 27]. In this work, we consider the case of the multiple second-order phase transitions, i.e., $\mathrm{SF} \rightarrow \mathrm{SS} \rightarrow$ DW. As we show, the system exhibits qualitatively different behavior in the resultant DW state depending on the value of $\tau_{\mathrm{Q}}$.

The practical protocol is the following;

$$
\frac{J_{c 1}-J(t)}{J_{c 1}}=\frac{J_{i}-J_{c 1}}{J_{c 1}} \frac{t}{\tau_{\mathrm{Q}}}, \quad t \in\left[-\tau_{\mathrm{Q}}, t_{f}\right],
$$

where $J_{i}=J\left(-\tau_{\mathrm{Q}}\right)$ is the initial value of $J(t)$, and we choose as $J_{i} / U=0.3\left(>J_{c 2} / U\right)$. At $t=0, J(0)=J_{c 1}$ and also we choose the final value as $J_{f}=J\left(t_{f}\right)=0$, i.e., the quench terminates at $t=t_{f}=\frac{J_{c 1}}{J_{i}-J_{c 1}} \tau_{\mathrm{Q}}$. See Fig. 8 , As the initial state, we use a GW-type wave function, in which small local fluctuations of the phase of $\left\{\Psi_{i}\right\}$ are added to the equilibrium $G W$ ground state. If we start the time evolution with the genuine SF state with a totally coherent phase, a DW-SF heterogeneous state forms as we observed in the previous work for the first-oder phase transition [27]. See Fig. (9) for the final state of the DW-SF heterogeneity. 

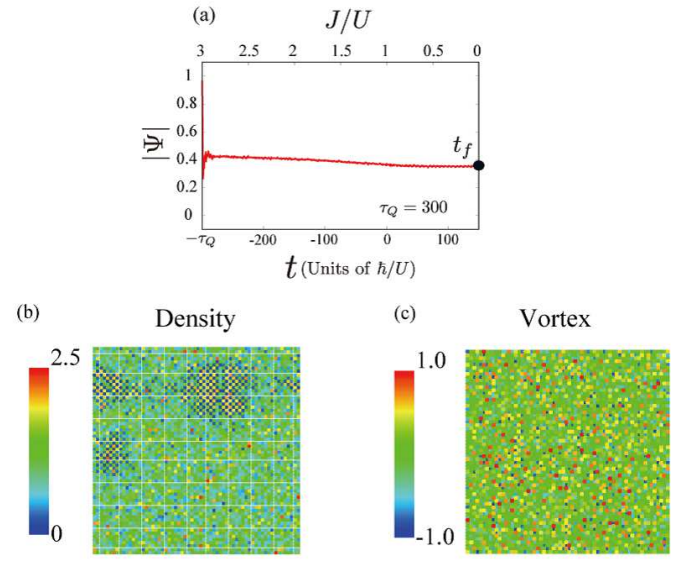

FIG. 9. If we start the time evolution with the genuine SF state with a totally coherent phase, a DW-SF heterogeneous state forms after crossing the DW phase transition. We show snapshots of the heterogeneous state of the DW and SF at $t=t_{f}$ that forms as a result of the evolution from the genuine SF state. Density profile exhibits clear formation of local DW regimes in the rather homogeneous background. Snapshot of vortices indicates that they proliferate.

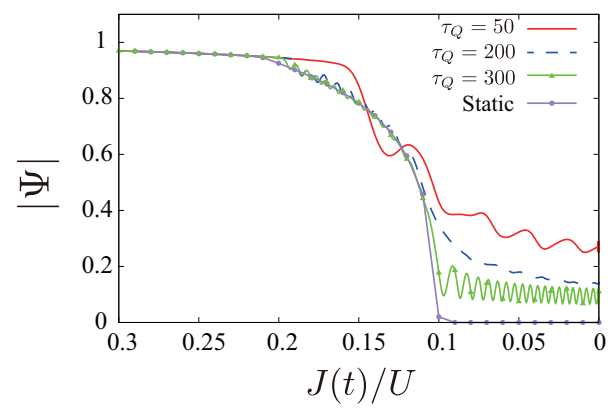

FIG. 10. SF amplitude as a function of $J / U$ for various quench times, $\tau_{\mathrm{Q}}$ 's. Results are compared with the equilibrium values.

We first show the SF amplitude $|\Psi|$ as a function of $J(t) / U$ in Fig. 10 for various quench times $\tau_{\mathrm{Q}}$ 's. As explained above, $J_{c 1} / U \simeq 0.10$ and $J_{c 2} / U \simeq 0.22$. For larger $\tau_{\mathrm{Q}}$, the results are getting closer to the static case as it is expected. However in all cases, the SF amplitude $|\Psi|$ has a finite value for $t \rightarrow t_{f}$.

It is also interesting to see density profile at $t=t_{f}$ for the above various $\tau_{\mathrm{Q}}$ 's. We show the obtained results in Fig. 11] For every $\tau_{\mathrm{Q}}$, there are domain walls separating DW regions, and for larger $\tau_{\mathrm{Q}}$, the less domain walls form. Close look at domain walls reveals that the pattern of the DW changes as crossing domain walls, and the expectation value of particle number at each site in domain walls fluctuates and takes a fractional value. This means that strong quantum fluctuations take place (a) $\tau_{Q}=50 \quad t_{f}=25$
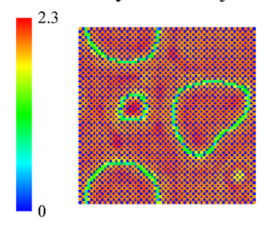

(b) $\tau_{Q}=200 t_{f}=100$

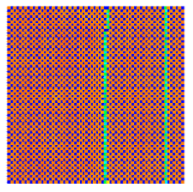

(c) $\tau_{Q}=300 \quad t_{f}=150$

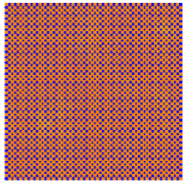

FIG. 11. Density profiles at $t=t_{f}$ for various quench times, $\tau_{\mathrm{Q}}$ 's. $J\left(t_{f}\right)=0$. Domain walls separating DW regions form and the total length of domain walls decreases as $\tau_{\mathrm{Q}}$ increases.
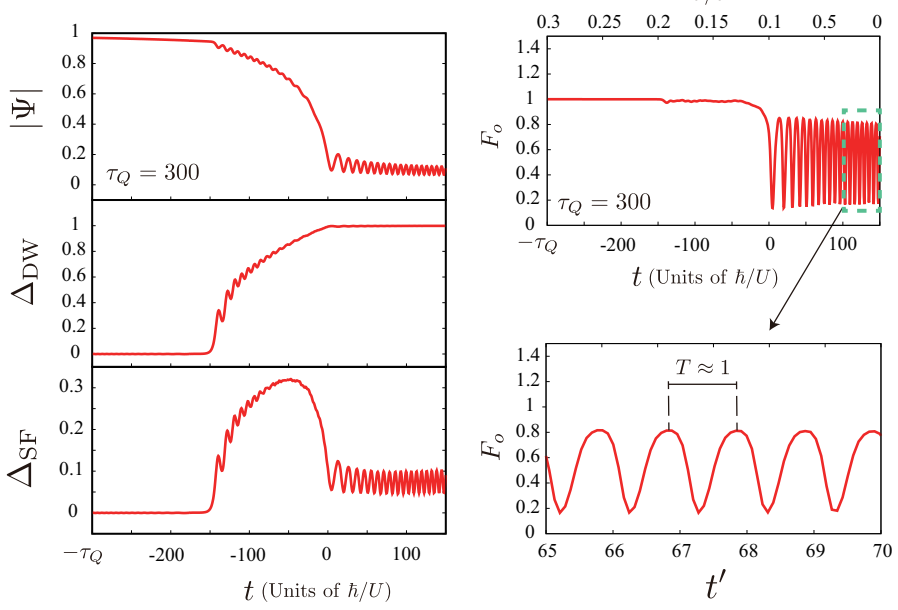

FIG. 12. Left panels: Physical quantities $|\Psi|, \Delta_{\text {DW }}$ and $\Delta_{\text {SF }}$ as a function of time for $\tau_{\mathrm{Q}}=300$. Right panels: First-order correlation $F_{o}$. After $t=0$, it exhibits the collapse-revival behavior. $t^{\prime} \equiv t \cdot \frac{U}{2 \pi} . \quad J(0) / U=J_{c 1} / U$ and $J(-150) / U \approx$ $J_{c 2} / U$.

inside of domain walls.

Next, physical quantities $|\Psi|, \Delta_{\mathrm{DW}}$ and $\Delta_{\mathrm{SF}}$ are shown in Fig. 12 as a function of time for $\tau_{\mathrm{Q}}=300 .|\Psi|, \Delta_{\mathrm{DW}}$ and $\Delta_{\mathrm{SF}}$ exhibit expected behaviors. In order to investigate a SF phase coherence in detail, we calculated the first-order correlation defined by

$$
F_{o}=\frac{1}{2 N_{s}} \sum_{i, j}\left(\left\langle a_{i}^{\dagger} a_{j}\right\rangle+\text { c.c. }\right) \text {. }
$$

In Fig. 12, we show the calculation of $F_{o}$ for $\tau_{\mathrm{Q}}=300$ as a function of time. After $t=0, F_{o}$ exhibits fluctuating behavior and close look at the oscillating regime shows that the period $T \approx 1$. In Fig. 13, we show $F_{o}$ as a function of time for $\tau_{\mathrm{Q}}=50,200$ and 300 . For ever quench time $\tau_{\mathrm{Q}}, F_{o}$ exhibits oscillating behavior after passing $t \approx 0$, but the pattern of oscillation strongly depends on $\tau_{\mathrm{Q}}$. This behavior may be related to the collapse-revival phenomenon that results from the surviving phase coherence of the $S F$ as studied in Refs. 34, 42 47]. In fact for the product of the genuine coherent state, $|\mathrm{SF}\rangle=\prod_{i}\left|\rho_{i}, \theta_{i}\right\rangle$ with $a_{i}\left|\rho_{i}, \theta_{i}\right\rangle=\sqrt{\rho_{i}} e^{i \theta_{i}}\left|\rho_{i}, \theta_{i}\right\rangle, F_{o}$ is calculated as follows 


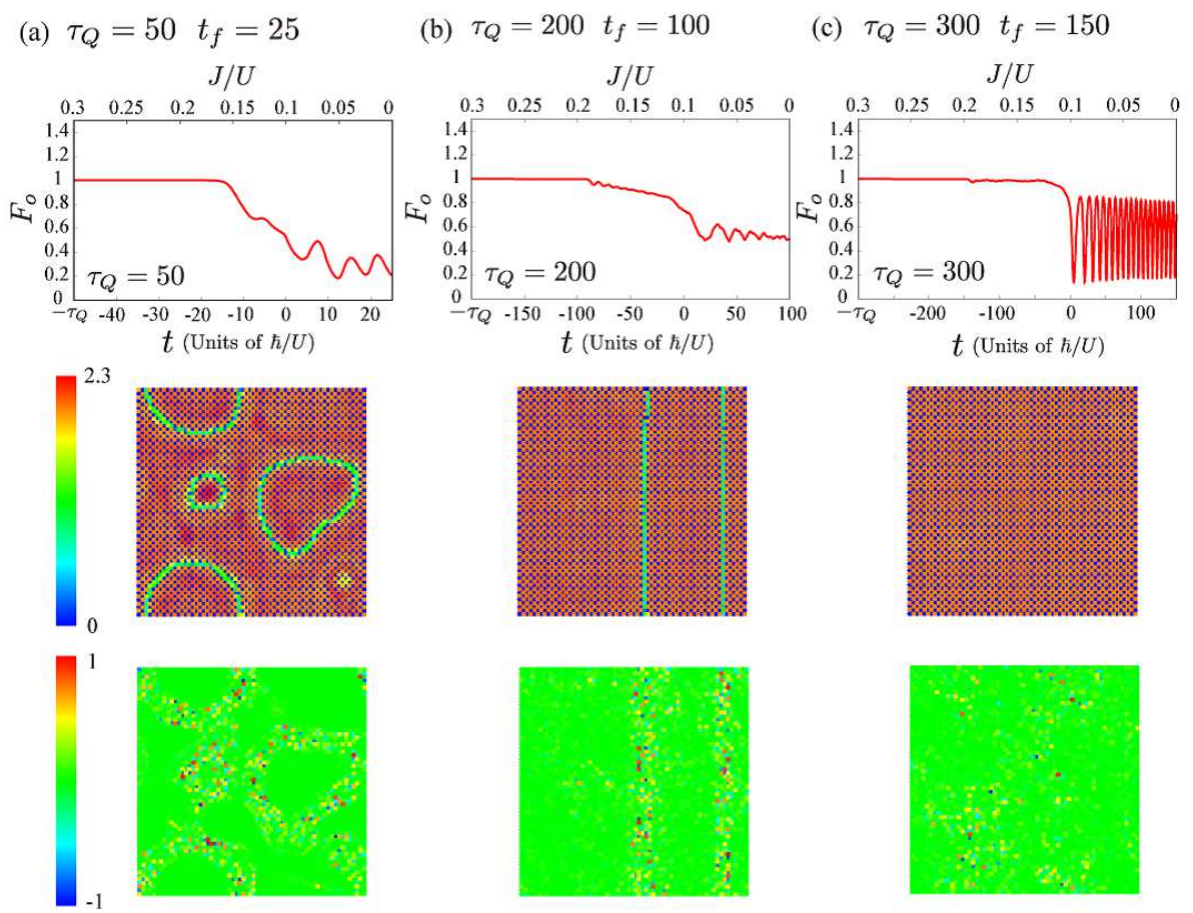

FIG. 13. Upper panel: First-order correlation function as a function of time for $\tau_{\mathrm{Q}}=50,200$ and $300 . t_{f}$ is the time at which the quench is terminated as shown in Fig. 8 Middle panel: Density profiles corresponding to the above times (Fig. 11). Lower panel: Vortex distributions corresponding to the above times. For $\tau_{\mathrm{Q}}=50$ and $\tau_{\mathrm{Q}}=200$, rather clear domain walls form although their shapes are different in the two cases. Vortices locate at the domain walls. On the other hand for $\tau_{\mathrm{Q}}=300$, locations of vortices are random.

[42],

$$
\begin{aligned}
& \left\langle\mathrm{SF}\left|e^{i H_{\mathrm{DW}} t} a_{i}^{\dagger} a_{j} e^{-i H_{\mathrm{DW} t}}\right| \mathrm{SF}\right\rangle \\
& \propto \sqrt{\rho_{i} \rho_{j}} e^{i\left(\theta_{j}-\theta_{i}\right)} \exp \left\{\rho_{i}\left(e^{i t U}-1\right)+\rho_{j}\left(e^{-i t U}-1\right)\right\} \\
& \times \exp \left\{\rho_{k}\left(e^{i t V}-1\right)+\rho_{\ell}\left(e^{-i t V}-1\right)\right\},
\end{aligned}
$$

where

$$
H_{\mathrm{DW}}=\frac{U}{2}\left\{n_{i}\left(n_{i}-1\right)+n_{j}\left(n_{j}-1\right)\right\}+V\left(n_{i} n_{k}+n_{j} n_{\ell}\right) .
$$

In the DW-type configurations such as $\rho_{i}, \rho_{j} \gg \rho_{k}, \rho_{\ell}$, the on-site $U$-term in Eq. (15) dominates over the NN $V$ term, and the oscillation period approximately is given by $2 \pi / U$. This explains the result in Fig. 12 .

In order to verify the above expectation, we study the cases of various $\tau_{\mathrm{Q}}$ 's, and show $F_{o}$ and vortex configurations for $J / U \approx 0$ in Fig. 13. For $\tau_{\mathrm{Q}}=50$ and 200 , rather clear domain walls exist, and interestingly enough, large amount of vortices reside on these domain walls. Therefore, the SF phase coherence is destroyed. On the other hand for $\tau_{\mathrm{Q}}=300$, existence of domain walls are not so clear, and the number of vortices is small and vortices seem locate rather randomly. We expect that this is the origin for the oscillating behavior of $F_{o}$. In summary, we observe that for slower quench from the SF to DW, the
SF amplitude $|\Psi|$ is getting smaller but the SF phase coherence is getting stronger compared to the faster quench as the vortex distribution and the first-order correlation $F_{o}$ indicate.

\section{CONCLUSION AND DISCUSSION}

In this paper, we studied the EBHM on the square lattice, which is expected to be realized by the ultra-cold atomic gases and quantum simulated. We first clarify the phase diagram of the system near the unit filling and $V / U=0.375$. There are three phases, the DW, SS and $\mathrm{SF}$. Then we studied the non-equilibrium quench dynamics by varying the hopping amplitude as a function of time.

In the quench dynamics from the DW to SS, we observed the time evolution of the SF amplitude and verified that it exhibits similar behavior in the Mott to SF second-order phase transition. The correlation length of the SF order, vortex density, $\hat{t}$ and $t_{\text {eq }}$, all exhibit the scaling laws with respect to the quench time $\tau_{\mathrm{Q}}$. By using the $\mathrm{KZ}$ scaling hypothesis, the values of critical exponents $\nu$ and $z$ were estimated from our numerical simulations, and we found that $z$ is close to the value of the 3D XY model but the estimated $\nu$ does not agree with the value of the 3D XY model. This discrepancy 
may stem from the NN repulsion and the DW order.

Next, we investigated the quench dynamics from the DW to SF through the SS. We verified that the phase degrees of freedom of the SF order parameter experiences the coarsening process as in the Mott to SF transition. The correlation length of the SF was also measured and we found that it gets large in the SF regime. This result implies that a SF at finite temperature forms as a result of the energy injection by the quench. On the other hand, the DW order smoothly decreases after passing the static transition point to the SS and vanishes at the transition to the SF.

Finally, we investigated the quench dynamics from the SF to DW. The SF amplitude starts to decrease at the SF-SS transition point $J_{c 2}$. After passing the SS-DW transition point, it exhibits the oscillating behavior for $\tau_{\mathrm{Q}}=300$. Observation of the first-order correlation of the SF indicates that it is nothing but the collapse-revival phenomenon of the quenched SF correlation in the DW regime. Similar phenomenon was discussed for the SFMott quench dynamics in the previous papers [26, 34, 42].

We hope that the phenomena that were investigated here will be observed in ultra-cold atomic experiments soon. ${ }^{168} \mathrm{Er}$ bosonic atom is a candidate for quantum simulation of the EHBM, as its dipole magnetic moment, $7 \mu_{\mathrm{B}}\left(\mu_{\mathrm{B}}=\right.$ the Bohr magneton), is fairly large. In the previous paper [48], we studied ${ }^{168} \mathrm{Er}$ systems on an optical lattice, and showed that the EBHM with $V / U \approx 0.3$ can be realized. Furthermore, some related experiments on ${ }^{168} \mathrm{Er}$ systems were performed and observation of a ground state with a DW order was reported [49].

Recently, there appeared very interesting theoretical study on universality in the dynamics of quench phase transition 50]. There, by using equations of motion or Ginzburg-Landau-type arguments, the KZ scaling was re-derived. In Ref. [27], this analysis was successfully applied to the first-order phase transition in the EBHM in the vicinity of the half filling. It is quite interesting to see how this approach is applied to the present multisecond-order phase transitions. This problem is under study, and results will be reported in a future publication.

\section{ACKNOWLEDGMENTS}

Y. K. acknowledges the support of a Grant-in-Aid for JSPS Fellows (No.17J00486).
[1] I. M. Georgescu, S. Ashhab, and F. Nori, Rev. Mod. Phys. 86, 153 (2014).

[2] J. I. Cirac and P. Zoller, Nat. Phys. 8, 264 (2012).

[3] I. Bloch, J. Dalibard, and W. Zwerger, Rev. Mod. Phys. 80, 885 (2008).

[4] M. Lewenstein, A. Sanpera, and V. Ahufinger, Ultracold Atoms in Optical Lattices: Simulating Quantum Manybody Systems (Oxford University Press, Oxford, 2012).

[5] K. Biedroń, M. Łacki, and J. Zakrzewski Phys. Rev. B 97, 245102 (2018).

[6] T. W. B. Kibble, J. Phys. A: Math. Gen. 9, 1387 (1976).

[7] T. W. B. Kibble, Phys. Rep. 67, 183 (1980).

[8] W. H. Zurek, Nature 317, 505 (1985).

[9] W. H. Zurek, Acta Phys. Pol. B 24, 1301 (1993).

[10] W. H. Zurek, Phys. Rep. 276, 177 (1996).

[11] See for example, A. del Campo and W. H. Zurek, Int. J. Mod. Phys. A 29, 1430018 (2014).

[12] N. Navon, A. L. Gaunt, R. P. Smith, and Z. Hadzibabic, Science 347, 167 (2015).

[13] J. Beugnon and N. Navon, J. Phys. B: At. Mol. Opt. Phys. 50, 022002 (2017).

[14] L. Chomaz, L. Corman, T. Bienaime, R. Desbuquois, C. Weitenberg, S. Nascimbene, J. Beugnon, and J. Dalibard, Nature Comm. 6, 6172 (2015).

[15] J. Dziarmaga, Phys. Rev. Lett. 95, 245701 (2005).

[16] A. Polkovnikov, Phys. Rev. B 72, 161201(R) (2005).

[17] W. H. Zurek, U. Dorner, and P. Zoller, Phys. Rev. Lett. 95, 105701 (2005).

[18] A. Chandran, A. Erez, S. S. Gubser, and S. L. Sondhi, Phys. Rev. B 86, 064304 (2012).

[19] J. Sonner, A. del Campo, and W.H. Zureck, Nature Communication 6, 7406 (2015).
[20] A. Francuz, J. Dziarmaga, B. Gardas, and W. H. Zurek, Phys. Rev. B 93, 075134 (2016).

[21] D. Chen, M. White, C. Borries, and B. DeMarco, Phys. Rev. Lett. 106, 235304 (2011).

[22] S. Braun, M. Friesdrof, S. S. Hodgman, M. Schreiber, J. P. Ronzheimer, A. Riera, M. del Rey, I. Bloch, J. Eisert, and U. Schneider, Proc. Natl. Acad. Sci. USA 112, 3641 (2015).

[23] M. Anquez, B. A. Robbins, H. M. Bharath, M. Boguslawski, T. M. Hoang, and M. S. Chapman, Phys. Rev. Lett. 116, 155301 (2016).

[24] L. W. Clark, L. Feng, and C. Chin, Science 354, 606 (2016).

[25] J-M. Cui, Y-F. Huang, Z-W. Wang, D-Y. Cao, J. Wang, W-M. Lv, L. Luo, A. del Campo, Y-J. Han, C-F. Li, and G-C. Guo, Sci. Rep. 6, 33381 (2016).

[26] K. Shimizu, Y. Kuno, T. Hirano, and I. Ichinose, Phys. Rev. A 97, 033626 (2018).

[27] K. Shimizu, T. Hirano, J. Park, Y. Kuno, and I. Ichinose, New J. Phys. 20, 083006 (2018)

[28] T. Kimura, Phys. Rev. A 84, 063630 (2011).

[29] T. Ohgoe, T. Suzuki, and N. Kawashima, Phys. Rev. B 86, 054520 (2012).

[30] L-J. Zhai, H-Y. Wang, and S. Yin, Phys. Rev. B 97, 134108 (2018).

[31] D. Jaksch, V. Venturi, J. I. Cirac, C. J. Williams, and P. Zoller, Phys. Rev. Lett. 89, 040402 (2002).

[32] J. Zakrzewski, Phys. Rev. A 71, 043601 (2005).

[33] M. Jreissaty, J. Carrasquilla, F. A. Wolf, and M. Rigol, Phys. Rev. A 84, 043610 (2011).

[34] M. Buchhold, U. Bissbort, S. Will, and W. Hofstetter, Phys. Rev. A 84, 023631 (2011). 
[35] S. S. Natu, K. R. A. Hazzard, and E. J. Mueller, Phys. Rev. Lett. 106, 125301 (2011).

[36] H. Fehrmann, M. A. Baranov, B. Damski, M. Lewenstein, and L. Santos, Opt. Commun. 243, 23 (2004).

[37] N. Horiguchi, T. Oka, and H. Aoki, Journal of Physics: Conference Series 150, 032007 (2009).

[38] M. Iskin, Phys. Rev. A 83, 051606 (R) (2011).

[39] P. M. Chesler, A. M. García-García, and H. Liu, Phys. Rev. X 5, 021015 (2015).

[40] Y. Kuno, K. Kataoka, and I. Ichinose, Phys. Rev. B 87, 014518 (2013).

[41] E. Burovski, J. Machta, N. Prokofev, and B. Svistunov, Phys. Rev. B 74, 132502 (2006).

[42] U. R. Fischer and B. Xiong, Phys. Rev. A 84, 063635 (2011).

[43] J. Dziarmaga, Adv. Phys. 59, 1063 (2010).
[44] S. Will, T. Best, U. Schneider, L. Hackermu, and I. Bloch, Nature 465, 197 (2010).

[45] M. Buchhold, U. Bissbort, S. Will, and W. Hofstetter, Phys. Rev. A 84, 023631 (2011).

[46] C. Kollath, A. M. Lauchli, and E. Altman, Phys. Rev. Lett. 98, 180601 (2007).

[47] F. Meinert, M. J. Mark, E. Kirilov, K. Lauber, P. Weinmann, M. Gröbner, and H.-C. Nägerl, Phys. Rev. Lett. 112, 193003 (2014).

[48] Y. Kuno, K. Shimizu, and I. Ichinose, Phys. Rev. A 95, 013607 (2017).

[49] S. Baier, M. J. Mark, D. Petter, K. Aikawa, L. Chomaz, Z. Cai, M. Baranov, P. Zoller, and F. Ferlaino, Science 352, 201 (2016).

[50] G. Nikoghosyan, R. Nigmatullin, and M. B. Plenio, Phys. Rev. Lett. 116, 080601 (2016). 